\title{
The energy distribution of electrons in radio jets
}

\author{
Alexandros Tsouros ${ }^{1}$ and Nikolaos D. Kylafis ${ }^{1,2}$ \\ 1 University of Crete, Physics Department \& Institute of Theoretical \& Computational Physics, 71003 Heraklion, Crete, Greece \\ e-mail: kylafis@physics.uoc.gr \\ 2 Foundation for Research and Technology-Hellas, IESL, 71110 Heraklion, Crete, Greece
}

Received 8 March 2017 / Accepted 13 June 2017

\begin{abstract}
Context. Black-hole and neutron-star X-ray binaries exhibit compact radio jets, when they are in the so called quiescent, hard, or hard intermediate states. The radio spectrum in these states is flat to slightly inverted, i.e., the spectral index of the observed flux density is in the range $0 \lesssim \alpha \lesssim 0.5$. It is widely accepted that the energy distribution of the electrons, in the rest frame of the jet, is a power law with index in the range $3 \lesssim p \lesssim 5$.

Aims. Contrary to what our thinking was decades ago, now we know that the jets originate in the hot, inner flow around black holes and neutron stars. So it is worth investigating the radio spectrum that is emitted by a thermal jet as a function of direction.

Methods. As an example, we consider a parabolic jet and, with the assumption of flux freezing, we compute the emitted spectrum in all directions, from radio to near infrared, using either a thermal distribution of electrons or a power-law one.

Results. We have found that parabolic jets with a thermal distribution of electrons give also flat to slightly inverted spectra. In particular, for directions along the jet $(\theta=0)$, both distributions of electron energies give $\alpha=0 \pm 0.01$. The index $\alpha$ increases as the viewing angle $\theta$ increases and for directions perpendicular to the jet $(\theta=\pi / 2)$, the thermal distribution gives $\alpha=0.40 \pm 0.05$, while the power-law distribution gives $\alpha=0.20 \pm 0.05$. The break frequency $v_{\mathrm{b}}$, which marks the transition from partially optically thick to optically thin synchrotron emission, is comparable for the power-law and the thermal distributions.

Conclusions. Contrary to common belief, it is not necessary to invoke a power-law energy distribution of the electrons in a jet to explain its flat to slightly inverted radio spectrum. A relativistic Maxwellian produces similar radio spectra. Thus, the jet may be the widely invoked "corona" around black holes in X-ray binaries.
\end{abstract}

Key words. accretion, accretion disks - X-rays: binaries - stars: jets - radio continuum: stars

\section{Introduction}

Black-hole X-ray binaries (BHXB) always exhibit a compact radio jet when they are in one of three spectral states: quiescent, hard, and hard intermediate (Fender et al. 2004, 2009; Fender \& Gallo 2014; Gallo et al. 2014). For a classification of the spectral states of BHXB see Belloni et al. (2005).

For the formation of the jet, two mechanisms have been proposed: plasma gun/magnetic tower (Contopoulos 1995; LyndenBell 1996) and centrifugal driving (Blandford \& Payne 1982). Both of them require a strong, large-scale, poloidal magnetic field. Such a magnetic field can either originate from a large distance from the black hole and the advecting flow carries it to the inner region and amplifies it (Igoumenshchev 2008; Lovelace et al. 2009; Tchekhovskoy et al. 2011), or it can be produced locally by the Cosmic Battery (Contopoulos \& Kazanas 1998; see also Contopoulos et al. 2006, 2009, 2015; Christodoulou et al. 2008). We favor the Cosmic Battery, because jets in BHXB are destroyed and re-created within hours, when the sources cross the so-called jet line (Fender et al. 2004; Miller-Jones et al. 2012). We consider it highly unlikely that the sources anticipate the destruction of their jet and its subsequent re-formation so as to "request" a magnetic field from far away, which should arrive at the inner part of the flow at the time that it is needed. Instead, we think that the observations require the strong poloidal magnetic field to be produced locally, at the right place and the right time. The formation and destruction of jets in the context of the Cosmic Battery, as well as the relevant timescales, have been discussed in Kylafis et al. (2012). An explanation of the rich phenomenology during an outburst of a BHXB has been offered by Kylafis \& Belloni (2015a,b).

The spectra of BHXB from the radio to the near infrared are flat to slightly inverted, i.e. flux density $S_{v} \propto v^{\alpha}$, with $0 \lesssim$ $\alpha \lesssim 0.5$ (Mirabel \& Rodriguez 1999; Fender et al. 2000, 2001; Fender 2001; Russell et al. 2006; Corbel et al. 2013; Russell \& Shahbaz 2014; Tetarenko et al. 2015). A characteristic frequency in this spectrum is the break frequency $v_{\mathrm{b}}$, where the partially optically thick jet becomes optically thin. This is an important frequency, because $S_{v_{\mathrm{b}}}$ is an indication of the total power emitted by the jet. The frequency $v_{\mathrm{b}}$ varies from source to source (Russell et al. 2013a) and also for the same source as a function of time (Russell et al. 2013b, 2014). Above $v_{\mathrm{b}}$, the jet is optically thin and its spectrum falls with an index $-1 \leq \alpha \leq-0.5$ (Fender 2001).

In 1979, synchrotron radio spectra were calculated for both, a Maxwellian distribution of electrons (Jones \& Hardee 1979) and for a power-law one Blandford \& Königl (1979). In subsequent years, the power-law model became the standard one and the research efforts concentrated on explaining how a power-law distribution of electron energies can be produced.

Two mechanisms can produce power-law distributions of electron energies: shocks (Heavens \& Meisenheimer 1987; for a review see Drury 1983) and magnetic reconnection (Spruit et al. 2001; Drenkhahn \& Spruit 2002; Sironi \& Spitkovsky 2014; Sironi et al. 2015; for a review see Kagan et al. 2015). The 
question then arises: are shocks and/or magnetic reconnection guarranteed to be present in the entire jet, from its base (where the frequency $v_{\mathrm{b}}$ is determined) to the top? One can envision shocks, due to an uneven flow in the jet, and magnetic reconnection, due to partially turbulent magnetic fields, but it is hard to imagine that these mechanisms operate in the entire jet.

In recent years, it has been accepted (Fender 2006; Fender \& Gallo 2014; Kylafis et al. 2012; Kylafis \& Belloni 2015a,b) that the jets in BHXB originate in the geometrically thick, optically thin, hot, inner flow around black holes, where the temperature of the electrons is large (hundreds of $\mathrm{keV}$; Ichimaru 1977; Narayan \& Yi 1994, 1995; Abramowicz et al. 1995; Blandford \& Begelman 1999; Narayan et al. 2000; Qataert \& Gruzinov 2000; Yuan et al. 2005). Outside the hot flow, the accretion disk is radiatively efficient and geometrically thin, i.e. Shakura-Sunyaev-type. The transition radius between the two types of flow decreases with increasing mass accretion rate and the Shakura-Sunyaev disk extends all the way to the inner stable circular orbit at high accretion rates, when the sources are in the so called soft state and no jet is present (Fender et al. 1999; Russell et al. 2011).

In this picture, it is natural to expect that the electrons in the jet, at least at its base, should be thermal or close to thermal. Therefore, even out of curiosity, we examine what type of radio spectrum is produced by a thermal distribution of electrons in the jet.

As mentioned above, most of the theoretical work on the radio emission from jets has assumed a power-law distribution of electron energies (see however Falcke \& Markoff 2000, who considered also a thermal jet model for Sgr A*). An extensive study of jet radio spectra using a power-law distribution was done by Kaiser (2006). An also extensive study, using both power-law and thermal distributions, was done by Pe'er \& Casella (2009). In order to obtain analytic results, both of these studies calculated spectra in the direction perpendicular to the jet $(\theta=\pi / 2)$. However, as we show below, the spectral index $\alpha$ depends strongly on the observation angle $\theta$.

In this Letter we consider a simple jet model, compute the radio spectrum as a function of $\theta$, and demonstrate that two completely different electron energy distributions (thermal and power-law) result in similar spectra. In subsequent work, we will explore different models to see if it is possible to infer the electron energy distribution from observations.

In Sect. 2 we describe our model, in Sect. 3 we compute the radio emission from the jet, in Sect. 4 we remark on some aspects of our calculations, and in Sect. 5 we present our conclusions.

\section{The model}

\subsection{Characteristics of the jet}

As a demonstration, we assume a parabolic jet, which has been used extensively before (see Sect. 4). In other words, we assume that the radius of the jet as a function of distance from the center of the compact object is

$R(z)=R_{0}\left(z / z_{0}\right)^{1 / 2}$

where $R_{0}$ is the radius at the base of the jet and $z_{0}$ is the height of the base of the jet.

For simplicity, we assume that the jet is accelerated close to its launching region and that it has constant velocity $v_{\|}=0.8 c$. From the continuity equation we infer that the number density of the electrons in the jet as a function of distance is

$n_{\mathrm{e}}(z)=n_{0} z_{0} / z$

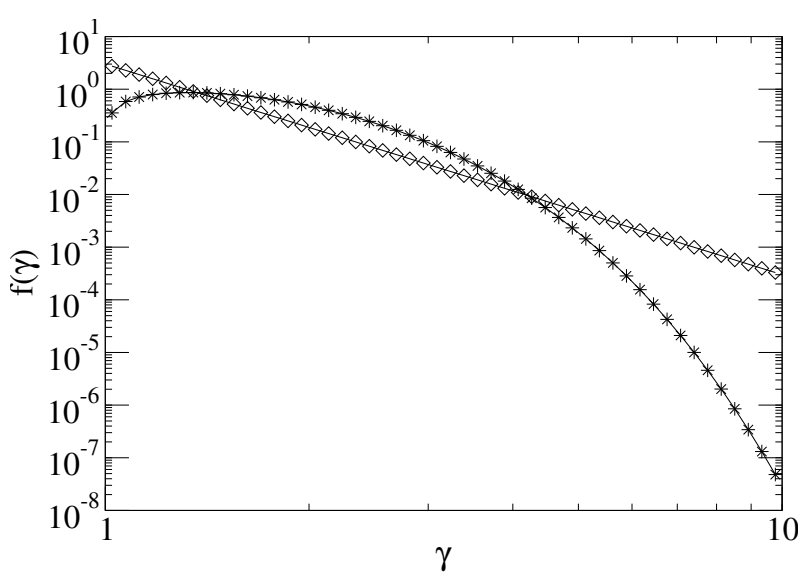

Fig. 1. Maxwell-Jüttner distribution (stars) for $\Theta=k T_{\mathrm{e}} / m_{\mathrm{e}} c^{2}=0.4$ and of the power-law distribution (diamonds) for $p=4$ and $\gamma_{\min }=1$, $\gamma_{\max }=10$.

where $n_{0}$ is the number density of the electrons at the base of the jet.

For the magnetic field in the jet, we also make the simple assumption that it is nearly parallel to the $z$-axis and that its strength is determined by flux conservation along the jet: $B(z) \pi R^{2}(z)=$ const. This implies that

$B(z)=B_{0} z_{0} / z$

where $B_{0}$ is the strength of the magnetic field at the base of the jet.

\subsection{Electron energy distribution}

For the energy distribution of the electrons in the rest frame of the jet, or equivalently for the distribution of the Lorentz factor $\gamma$ since $E_{\mathrm{e}}=\gamma m_{\mathrm{e}} c^{2}$, we assume either a relativistic Maxwellian, i.e. a Maxwell-Jüttner distribution,

$f_{\mathrm{MJ}}(\gamma)=\frac{\gamma^{2} \beta}{\Theta K_{2}(1 / \Theta)} \mathrm{e}^{-\gamma / \Theta}$,

where $\beta=\sqrt{1-1 / \gamma^{2}}, \Theta=k T_{\mathrm{e}} / m_{\mathrm{e}} c^{2}$, and $K_{2}$ is the modified Bessel function of the second kind, or a power-law distribution

$f_{\mathrm{pl}}(\gamma)=\frac{p-1}{\gamma_{\min }^{-p+1}-\gamma_{\max }^{-p+1}} \gamma^{-p}$,

in the range $\gamma_{\min } \leq \gamma \leq \gamma_{\max }$.

An analytic treatment of cooling in a jet was described in Kaiser (2006). For the values of the parameters that we use, the synchrotron timescale at, say $z=10 z_{0}$, is about $1 \mathrm{~s}$, while the flow timescale there is $0.003 \mathrm{~s}$. Thus, synchrotron cooling can be neglected. For simplicity, we also neglect diabatic expansion cooling, because $\gamma(z) \propto\left(z_{0} / z\right)^{1 / 3}$ (Pe'er \& Casella 2009).

In Fig. 1 we plot the Maxwell-Jüttner distribution for $\Theta=0.4$ as a line with stars and a power law distribution with $p=4$ from $\gamma_{\min }=1$ to $\gamma_{\max }=10$ as a line with diamonds. Both distributions are normalized to unity. Despite the fact that the distributions described by Eqs. (4a) and (4b) are qualitatively different, in the range $1 \lesssim \gamma \lesssim 6$ the two distributions exhibit only quantitative differences.

The distribution of electrons as a function of $z$ and $\gamma$ is then

$n_{\mathrm{e}}(z, \gamma)=n_{\mathrm{e}}(z) f(\gamma)$,

where $n_{\mathrm{e}}(z)$ is given by Eq. (2) and $f(\gamma)$ is given by Eqs. (4a) or (4b). 


\subsection{Radiative transfer}

Ignoring possible Compton upscattering in the jet (for this the reader is referred to Reig et al. 2003; Giannios et al. 2004; Giannios 2005; Kylafis et al. 2008; Reig \& Kylafis 2015, 2016), the equation for the transfer of radio photons in the jet in direction $\hat{n}$, along which length is measured by $s$, is given by

$\frac{\mathrm{d} I(v, s)}{\mathrm{d} s}=j(v, s)-a(v, s) I(v, s)$

where $j(v, s)$ and $a(v, s)$ are the emission and absorption coefficients, respectively.

For mildly relativistic magnetic plasma, the gyrosychrotron emission formalism is the appropriate one. For simplicity, here we use the extreme relativistic formalism, in which the emission coefficient is (see Eq. (6.36) of Rybicki \& Lightman 1979)

$j(v, z)=n_{\mathrm{e}}(z) D(z) \int_{\gamma_{\min }}^{\gamma_{\max }} F\left(\frac{v}{v_{\mathrm{c}}}\right) f(\gamma) \mathrm{d} \gamma$,

where

$D(z)=\frac{\sqrt{3} e^{3} B(z) \sin \phi}{m_{\mathrm{e}} c^{2}}$,

$e$ is the charge of the electron, $\phi$ is the pitch angle of the electron,

$v_{\mathrm{c}}=\frac{3 \gamma^{2} e B(z) \sin \phi}{4 \pi m_{\mathrm{e}} c}$,

and

$F(x)=x \int_{x}^{\infty} K_{\frac{5}{3}}(u) \mathrm{d} u$,

where $K_{\frac{5}{3}}$ is the modified Bessel function of the second kind.

The absorption coefficient is (see Eq. (6.50) of Rybicki \& Lightman 1979)

$a(v, z)=-\frac{n_{\mathrm{e}}(z) D(z)}{8 \pi m_{\mathrm{e}} v^{2}} \int_{\gamma_{\min }}^{\gamma_{\max }} F\left(\frac{v}{v_{\mathrm{c}}}\right) \gamma^{2} \frac{\partial}{\partial \gamma}\left[\frac{f(\gamma)}{\gamma^{2}}\right] \mathrm{d} \gamma$

The formal solution to the radiative transfer Eq. (6), for a distant observer, is

$I(v)=\int_{s_{\mathrm{b}}}^{s_{\mathrm{f}}} j(v, s) \mathrm{d} s \mathrm{e}^{-\int_{s}^{s_{\mathrm{f}}} a\left(v, s^{\prime}\right) \mathrm{d} s^{\prime}}$,

where $s_{\mathrm{b}}$ and $s_{\mathrm{f}}$ are the back and the front intercepts of the line of sight with the surface of the jet.

The radio spectra of jets are observed to have a characteristic break frequency $v_{\mathrm{b}}$ (see Pe'er \& Casella 2009, for other possible characteristic frequencies), which is determined by the condition that the jet has optical depth equal to one at its base

$a\left(v_{\mathrm{b}}, z_{0}\right) R_{0}=1$,

where $R_{0}$ is the radius of the jet at its base (see Eq. (1)). This means that the entire jet is optically thin. Due to contamination of the radio spectrum, $v_{\mathrm{b}}$ may not be always evident in the observed spectra.

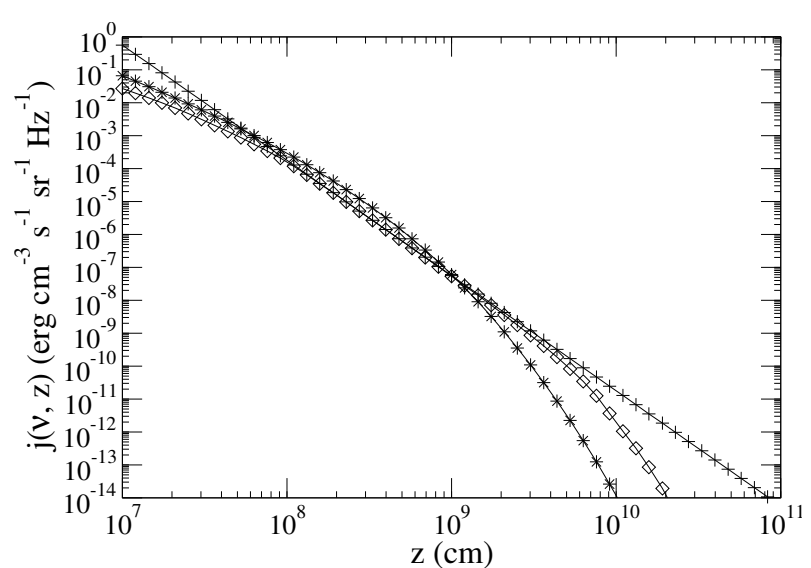

Fig. 2. Emission coefficient $j(v, z)$ as a function of $z$ for $v=10^{11} \mathrm{~Hz}$. The electron energy distributions are: thermal (stars), power-law with $1 \leq \gamma \leq \infty$ (plusses), and power-law with $1 \leq \gamma \leq 10$ (diamonds).

\section{Radio emission from the jet}

For the purposes of a demonstrative calculation, we assign reference values to the involved parameters. Thus, we take $R_{0}=$ $100 r_{\mathrm{g}}$, where $r_{\mathrm{g}}=15 \mathrm{~km}$ is the gravitational radius, $z_{0}=5 r_{\mathrm{g}}$, $n_{0}=10^{16} \mathrm{~cm}^{-3}, B_{0}=2 \times 10^{5} \mathrm{G}, \Theta=0.4, p=4, \gamma_{\min }=1$, and $\gamma_{\max }=\infty$. For the pitch angle we take the value $\phi=30$ degrees.

For the reference values of the parameters we find using Eq. (10) that $v_{\mathrm{b}}=2.7 \times 10^{14} \mathrm{~Hz}$ for the power-law distribution of electron energies and $v_{\mathrm{b}}=10^{14} \mathrm{~Hz}$ for the thermal distribution. If we restrict the power-law distribution to $\gamma_{\max }=10$, then $v_{\mathrm{b}}=1.6 \times 10^{14} \mathrm{~Hz}$.

In Fig. 2 we show the emission coefficient $j(v, z)$ as a function of $z$, for frequency $v=10^{11} \mathrm{~Hz}$. The symbols are: stars (thermal) and plusses (power-law). If we restrict the power-law distribution to $\gamma_{\max }=10$, then the corresponding line becomes that with the diamonds. It is evident from Fig. 2 that a power-law distribution with $\gamma_{\min }=1$ and $\gamma_{\max }=10$ has essentially the same emission coefficient as a thermal distribution.

We have solved numerically expression (9) and, for the reference values of the parameters, we have found the following values for the spectral index: $\alpha=0$ for both electron energy distributions if the viewing angle $\theta=0$. For $\theta=\pi / 2$, we have found $\alpha=0.4$ for the thermal distribution and $\alpha=0.2$ for the powerlaw distribution, the last one in agreement with Giannios (2005). If we restrict the power-law distribution to $1 \leq \gamma \leq 10$, then we find $\alpha=0.4$, something that was expected in view of the similarity of the emission coefficient for the two distributions. The value of $\alpha$ for both distributions increases smoothly from $\theta=0$ to $\theta=\pi / 2$. The variation of $\alpha$ with $\theta(\Delta \alpha=0.4)$ is much larger than the variation $(\Delta \alpha= \pm 0.05)$ caused by $0.2 \leq \Theta \leq 0.5$ or $3 \leq p \leq 5$. Also, there is no need to examine directions $\pi / 2<\theta \leq \pi$, because the approaching lobe of the jet dominates the emission.

\section{Discussion}

Models of BHXB invoke a thermal corona near the black hole to explain the observed hard X-ray spectrum by thermal Comptonization(see Done et al. 2007, for a review). Here, we have demonstrated that a thermal jet is an excellent "corona" for the upscattering of soft photons. In fact, Comptonization in the jet can explain not only the observed power-law high-energy spectral index $\Gamma$ and the high-energy cutoff $E_{\mathrm{c}}$, but also a) the depedence of the time lag on Fourier frequency (Reig et al. 2003), 
b) the narrowing of the autocorrelation function with increasing photon energy (Giannios et al. 2004), c) the dependence of $\Gamma$ on either the time lag or the Lorentzian peak frequency (Kylafis et al. 2008), and d) the relation between $E_{\mathrm{c}}$ and phase lag (Reig $\&$ Kylafis 2015). It is very interesting that all the above correlations are explained with the same, simple, jet model, that has been used here as an example. In fact, this was one of the reasons for choosing this example.

\section{Conclusions}

Contrary to common belief that the flat to slightly inverted radio spectra from jets imply a power-law energy distribution of the electrons in the jet, we have demostrated here that a thermal distribution of electron energies produces essentially identical radio spectra.

We find it interesting that the index $\alpha$ of the radio spectrum of the outbursting source MAXI J1836-194 flip-flops between $\sim 0.2$ and $\sim 0.5$ (Russell et al. 2014). It is too early to infer that the energy distribution of the electrons alternates between thermal and power law, but it is intriguing.

Acknowledgements. We thank the anonymous referee for useful comments and suggestions. We also thank Dimitrios Giannios for useful discussions and comments. One of us (NDK) thanks Tomaso Belloni, Rob Fender, Sera Markoff, and Dave Russell for useful discussions.

\section{References}

Abramowicz, M. A., Chen, X., Kato, S., Lasota, J.-P., \& Regev, O. 1995, ApJ, 438, L37

Belloni, T., Homan, J., Casella, P., et al. 2005, A\&A, 440, 207

Blandford, R. D., \& Begelman, M. C. 1999, MNRAS, 303, L1

Blandford, R. D., \& Königl, A. 1979, ApJ, 232, 34

Blandford, R. D., \& Payne D. G. 1982, MNRAS, 199, 883

Christodoulou, D. M., Contopoulos, I., \& Kazanas, D. 2008, ApJ, 674, 388

Contopoulos, I. 1995, ApJ, 450, 616

Contopoulos, I., \& Kazanas, D. 1998, ApJ, 508, 859

Contopoulos, I., Kazanas, D., \& Christodoulou, D. M. 2006, ApJ, 652, 1451

Contopoulos, I., Christodoulou, D. M., Kazanas, D., \& Gabuzda, D. C. 2009, ApJ, 702, L148

Contopoulos, I., Nathanail, A., \& Katsanikas, M. 2015, ApJ, 805, 105

Corbel, S., Aussel, H., Broderick, J. W., et al. 2013, MNRAS, 431, L107

Done, C., Gierliński, M., \& Kubota, A. 2007, A\&ARv, 15, 1

Drenkhahn, G., \& Spruit, H. C. 2002, A\&A, 391, 1141

Drury, L. O'C. 1983, Rep. Prog. Phys., 46, 973

Falcke, H., \& Markoff, S. 2000, A\&A, 362, 113

Fender, R. P. 2001, MNRAS, 322, 31
Fender, R. P. 2006, in Compact Stellar X-ray Sources, eds. W. H. G. Lewin, \&

M. van der Klis (Cambridge University Press), 381

Fender, R., \& Gallo, E. 2014, Space Sci. Rev., 183, 323

Fender, R., Corbel, S., Tzioumis, T., et al. 1999, ApJ, 519, L165

Fender, R. P., Pooley, G. G., Durouchoux, P., Tilanus, R. P. J., \& Brocksopp, C. 2000, MNRAS, 312, 853

Fender, R. P., Hjellming, R. M., Tilanus, R. P. J., et al. 2001, MNRAS, 322, L23

Fender, R. P., Belloni, T. M., \& Gallo, E. 2004, MNRAS, 355, 1105

Fender, R. P., Homan, J., \& Belloni, T. M. 2009, MNRAS, 396, 1370

Gallo, E., Miller-Jones, J. C. A., Russell, D. M., et al. 2014, MNRAS, 445, 290

Giannios, D. 2005, A\&A, 437, 1007

Giannios, D., Kylafis, N. D., \& Psaltis, D. 2004, A\&A, 425, 163

Heavens, A. F., \& Meisenheimer, K. 1987, MNRAS, 225, 335

Ichimaru, S. 1977, ApJ, 214, 840

Igoumenshchev, I. V. 2008, ApJ, 677, 317

Jones, T. W., \& Hardee, P. E. 1979, ApJ, 228, 268

Kagan, D., Sironi, L., Cerutti, B., \& Giannios, D. 2015, SSRv, 191, 545

Kaiser, C. R. 2006, MNRAS, 367, 1083

Kylafis, N. D., \& Belloni, T. 2015a, Astrophys. Space Sci. Lib., 414, 245

Kylafis, N. D., \& Belloni, T. 2015b, A\&A, 574, A133

Kylafis, N. D., Papadakis, I. E., Reig, P., Giannios D., \& Pooley, G. G. 2008, A\&A, 489, 481

Kylafis N. D., Contopoulos, I., Kazanas, D., \& Christodoulou, D. M. 2012, A\&A, 538, A5

Lovelace, R. V. E., Rothstein, D. M., \& Bisnovatyi-Kogan, G. S. 2009, ApJ, 701, 885

Lynden-Bell, D. 1996, MNRAS, 279, 389

Miller-Jones, J. C. A., Sivakoff, G. R., Altamirano, D., et al. 2012, MNRAS, 421, 468

Mirabel, I. F., \& Rodriguez, L. F. 1999, ARA\&A, 37, 409

Narayan, R., \& Yi, I. 1994, ApJ, 428, L13

Narayan, R., \& Yi, I. 1995, ApJ, 452, 710

Narayan, R., Igumenshchev, I. V., \& Abramowicz, M. A. 2000, ApJ, 539, 798

Pe'er, A., \& Casella, P. 2009, ApJ, 699, 1919

Quataert, E., \& Gruzinov, A. 2000, ApJ, 539, 809

Reig, P., \& Kylafis, N. D. 2015, A\&A, 584, A109

Reig, P., \& Kylafis, N. D. 2016, A\&A, 591, A24

Reig, P., Kylafis, N. D., \& Giannios, D. 2003, A\&A, 403, L15

Russell, D. M., \& Shahbaz, T. 2014, MNRAS, 438, 2083

Russell, D. M., Fender, R. P., Hynes, R. I., et al. 2006, MNRAS, 371, 1334

Russell, D. M., Miller-Jones, J. C. A., Maccarone, T. J., et al. 2011, ApJ, 739, L19

Russell, D. M., Markoff, S., Casella, P., et al. 2013a, MNRAS, 429, 815

Russell, D. M., Russell, T. D., Miller-Jones, J. C. A., et al. 2013b, ApJ, 768, L35

Russell, T. D., Soria, R., Miller-Jones, J. C. A., et al. 2014, MNRAS, 439, 1390

Rybicki, G. B., \& Lightman, A. P. 1979, Radiative Processes in Astrophysics (New York: Wiley)

Sironi, L., \& Spitkovsky, A. 2014, ApJ, 783, L21

Sironi, L., Petropoulou, M., \& Giannios, D. 2015, MNRAS, 450, 183

Spruit, H. C., Daigne, F., \& Drenkhahn, G. 2001, A\&A, 369, 694

Tchekhovskoy, A., Narayan, R., \& McKinney, J. C. 2011, MNRAS, 327, L79

Tetarenko, A. J., Sivakoff, G. R., Miller-Jones, J. C. A., et al. 2015, ApJ, 805, 30

Yuan, F., Cui, W., \& Narayan, R. 2005, ApJ, 620, 905 